\title{
Race and place: complex constructs in breast cancer care
}

\author{
Emily M. Ray ${ }^{1,2}$, Stephanie B. Wheeler ${ }^{3,4}$, Katherine E. Reeder-Hayes ${ }^{1,3}$ \\ ${ }^{1}$ Division of Hematology/Oncology, Department of Medicine, ${ }^{2}$ Cecil G. Sheps Center for Health Services Research, ${ }^{3}$ Lineberger Comprehensive \\ Cancer Center, University of North Carolina, Chapel Hill, NC, USA; ${ }^{4}$ Department of Health Policy and Management, Gillings School of Global \\ Public Health, University of North Carolina, Chapel Hill, NC, USA \\ Correspondence to: Emily M. Ray, MD, MPH. UNC Division of Hematology and Oncology, Physicians Office Building, $3^{\text {rd }}$ floor, 170 Manning Drive, \\ CB 7305, Chapel Hill, NC 27599, USA. Email: Emily.ray@unchealth.unc.edu. \\ Comment on: Moore JX, Royston KJ, Langston ME, et al. Mapping hot spots of breast cancer mortality in the United States: place matters for Blacks \\ and Hispanics. Cancer Causes Control 2018. [Epub ahead of print].
}

Submitted Sep 19, 2018. Accepted for publication Sep 25, 2018.

doi: $10.21037 /$ tcr.2018.09.11

View this article at: http://dx.doi.org/10.21037/tcr.2018.09.11

Breast cancer is the most common cancer and the second leading cause of cancer deaths among women in the United States (1). Although cancer mortality has declined due to improvements in early detection and treatment, and the 5 -year overall survival is high $(89 \%)$, vulnerable patient groups including racial and ethnic minorities continue to experience disproportionate burdens of mortality from breast cancer (1). A growing body of literature supports longstanding concerns that racial, ethnic, geographic, and socioeconomic factors influence access and quality at all phases of oncologic care, from screening to treatment to surveillance to end-of-life care (2).

Black and other minority women have historically had lower rates of screening mammography compared to nonHispanic White (NHW) women (3), although recent studies show that this disparity in screening no longer exists (1). Importantly, Black and Hispanic women have higher risk of delays in breast cancer treatment (4), and Black women receive lower rates of gene expression profiling, which can aid in chemotherapy decision making (5). Black women, at least in urban areas, are more likely to have treatment delays or early termination of adjuvant chemotherapy (6) and minority women with early breast cancers are less likely to receive guideline-recommended radiation therapy, adjuvant chemotherapy, or endocrine therapy $(7,8)$. Black women also experience lower rates of adherence to adjuvant endocrine therapy (9). Despite a higher incidence of breast cancer among NHW women, mortality is higher and 5-year survival is lower among Black women (1). Further, Black women with breast cancer more often experience adverse financial outcomes than NHW women, including lost income, job loss, private insurance loss, cost-related delays or omission of care, and transportation-related delays or omission of care (10).

While Hispanics have lower incidence of breast cancer than other groups in the United States, these numbers continue to rise. ${ }^{1}$ Hispanics are more likely than their NHW counterparts to have late-stage disease at diagnosis, perhaps related to decreased screening mammography in the Hispanic population $(1,11)$. Hispanics in higher-segregation areas ("Hispanic neighborhoods") have lower incidence of breast cancer compared to Hispanics in more integrated communities (11), but present with more advanced cancers at diagnosis (12) and have higher breast cancer mortality (13). Lower incidence does not necessarily mean that there is less breast cancer among the Hispanic population, but instead may reflect lower rates of detection in the context of lower rates of screening. All-cause mortality among Hispanic women with breast cancer is much better than NHW or Black women with mortality rates of 47.7 per 100,000 in 2004 , compared to 74.0 and 92.4 among White and Black women, respectively. Contrastingly, when 5 -year cancer-specific mortality is examined, the probability of death in Hispanics exceeds that of NWH women (13.1\% vs. $11.1 \%)$ (11). Important for patient outcomes, foreign-born Hispanics are less likely to receive supportive care medications despite higher rates of reported pain, depression, and other supportive care needs (14).

The relationship between breast cancer epidemiology 
and geography is equally complex. Women with lower income, with lower education, and in rural areas receive screening mammography at lower rates, although screening rates increase when patients have insurance and no out-ofpocket cost for screening $(15,16)$. Breast cancer incidence is higher in urban areas compared to rural areas, in part mediated by higher socioeconomic status (SES) and higher density of primary care providers leading to increased rates of detection (17). Although women in urban areas experience higher incidence of breast cancer, women in rural areas are more likely to present with late-stage disease, again likely reflective of delayed diagnosis in the context of low uptake of screening mammography (18). Additionally, perhaps due to higher rates of poverty, lower rates of education, and decreased access to care, people in rural regions experience lower rates of initiation and adherence to adjuvant endocrine therapy (9) and worse cancer outcomes $(19,20)$.

Location or setting of care can modulate some of the documented racial disparities in breast cancer care. Minority breast cancer survivors have lower rates of recommended surveillance mammography (21); however, in systems with equal access to care, such as the military, minority women are equally or more likely to undergo surveillance mammography than NHW women (22). Interestingly, the racial disparities in breast cancer treatment seen in urban areas were not seen in one study of a rural region in Georgia (23). These findings suggest that race does not act as a solitary determinant of access to care but rather serves as one factor in a complex web of health determinants including geography, SES, social support, insurance status, and biology.

These disparities in access to and quality of care, as well as biologic and genetic differences, drive differences in breast cancer outcomes along racial and geographic lines. In the context of these known racial and geographic disparities in breast cancer, the study by Moore et al. looking at hot spots of breast cancer mortality in the United States (24) has several important implications. First, the authors provide a well-executed, national snapshot to demonstrate that both race and place clearly matter in determining breast cancer outcomes. Second, the work reminds us that all disparities are local. By this, we mean that an understanding of the local context is essential to interpreting racial differences in health outcomes, particularly in the context of cancer, which requires a complex network of local services for its diagnosis, treatment, and long-term follow-up. Finally, this paper raises interesting questions regarding the "right" way to approach geographic hot-spot analyses as they relate to the goal of eliminating racial disparities in cancer outcomes. Here, we will explore the findings of this paper and its implications.

\section{Race and place matter}

Race and geography are key determinants of health, and together they play a significant role in determining breast cancer treatment and outcomes. Two illustrative examples center around two regions with some of the highest poverty rates and lowest education in the United States-the Mississippi Delta and Appalachia. In the Mississippi Delta, Blacks suffer from higher rates of cancer mortality than Whites living in the same region and have higher mortality than Blacks living in other regions. While rural Appalachia is considered to be a similarly disadvantaged region in terms of accepted socioeconomic metrics and has higher cancer mortality than other regions of the United States (19); however, when compared to the Mississippi Delta, this region actually has lower cancer mortality (20).

In their study, Moore et al. found that specific regions of the United States were associated with a concentration of breast cancer mortality "hot spots" for Blacks and Hispanics, but they did not identify definitive geographic patterns in breast cancer mortality for NHW women. The use of novel geospatial methods, using an aggregation of three separate spatial clustering methods to identify hot spot counties for breast cancer mortality, and stratification of mortality rates by race/ethnicity, enabled the authors to characterize the interplay between race and place as determinants of health in a novel fashion. The study identified two hot spot areas for breast cancer mortality among Black women, both in the southeastern United States. For Hispanic women, two regions were identified, one in the southwestern region and one in central to southern Florida (24). Although not definitive, these findings suggest that geographic location may have greater impact on breast cancer mortality for minority than for NHW patients, and interestingly, that regions of the country with higher representation of particular racial/ethnic minority groups (the South for Black patients and the Southwest and southern Florida for Hispanic patients) may also harbor hot spots of higher mortality for those groups. Further, these findings suggest that dedicated resources and targeted approaches to improve breast cancer screening, diagnosis, treatment, and surveillance in particular locales, rather than targeting one racial/ethnic group across a wider geographic catchment, may have the greatest population-level impact on outcome 
disparities for Black and Hispanic women.

\section{All disparities are local}

Health disparities do not arise in a vacuum, and reducing health disparities requires an understanding of the complex socioeconomic and geographic setting in which disparities exist. In their paper, Moore et al. evaluate whether observed geographic differences are explained by county-level characteristics. They found that hot spot counties for all women had higher adult obesity, lower education, lower income, higher physical inactivity, and higher unemployment. Hot spot counties also had higher proportions of non-Hispanic Blacks and lower proportions of Hispanics and NHW (24) In other words, geographic locations associated with poor breast cancer mortality are, perhaps not surprisingly, also poor environments in terms of the basic social determinants of health, and these unhealthful environments are also majority-minority communities. Given these findings, it is reasonable to hypothesize that these breast cancer mortality hot spots likely co-occur with disproportionate burdens of other deadly diseases common in minority patients and adversely impacted by social determinants of health such as cardiovascular disease and diabetes. We are increasingly aware that obesity, and potentially chronic environmental stress (25), contribute to sub-optimal breast cancer outcomes, and that a high burden of co-morbid disease complicates the receipt of appropriate cancer therapies and survivorship care (26). Thus, the improvement of outcomes and eradication of disparities in breast cancer hot spots may relate as much to improvement of overall living conditions as to focused intervention on some specific aspect of cancer care delivery.

A deeper, more granular understanding of the characteristics and culture of these hot spot regions is required to interpret these disparities and find ways to reduce them. A local lens is required, and a one-size-fitsall approach is unlikely to impact breast cancer mortality in all of these hot spot counties. For example, community engagement through local churches may be more effective in the rural South, where the church is a key part of the fabric of society, than in the urban Northwest where such religious ties are not as closely held. Local knowledge and community engagement are required to understand the drivers of health disparities and areas for possible intervention. This underscores the importance of local and regional public health initiatives, concurrent with national- level changes, to reduce disparities and improve health for all.

\section{Defining the right lens}

Moore et al. use the approach of race-stratified analyses to explore one scientific question within the race-placeoutcome triad: whether patterns of geographic variation in breast cancer outcomes are similar across races, or whether the relationship of geography to breast cancer outcome varies by race. This question is important, and the Moore study is useful in demonstrating that the link between geography and breast cancer outcome likely functions differently depending on the race/ethnicity of the patient, with more variability by region for Black and Hispanic patients. However, the greater point of the study should not be lost in the details of the race-stratified analysis: overall, hot spots of breast cancer mortality in the United States are very likely to be in Southern states ( $72 \%$ of hot spots in this study), which have higher Black populations, poverty levels, and rates of co-morbidity. Likewise, hot spots were disproportionately located in communities of color which also suffered low levels of education, employment, and insurance as well as an adverse profile of body composition and physical activity. The implication of this finding for cancer disparities researchers is clear: while each community is unique, and while targeted local interventions are called for, we are unlikely to see a dramatic turn-around in the national racial mortality gap for breast cancer if we ignore the fundamental social causes of poor health and barriers to healthcare access with which minority breast cancer patients, as well as other patients, in "hot spot" communities must contend.

Two further points bear discussing. First, the authors of the Moore study define hot spots using a conservative statistical approach of co-identification by each of three geo-spatial analytic methods (Getic Ord, LISA, and Bayes method) and using a traditional threshold of $\mathrm{P} \leq 0.05$ to designate statistical significance. These methods designated approximately $2.5 \%$ of geographic locations as "hot spots". While this approach is sound and appropriate, application to intervention and policy decisions may require different thresholds, depending on the availability of resources and the size of the geographic area under consideration for intervention, which might be a state, region or other boundary, to define a scope that is feasible and pragmatic. Second, while race stratification is helpful in understanding geographic patterns in outcome within a specific use of resources, unstratified analyses are the most appropriate 
approach to identify hot spots for further study and intervention efforts, since the greatest impact on breast cancer mortality at the population level will be reaped from targeting the highest mortality areas, regardless of race.

\section{Heeding the call}

Understanding that race and place matter in cancer outcomes, that all disparities are local, and that race is a social construct with direct implications for health, public health researchers must develop interventions aimed at existing disparities. Moore and colleagues contribute valuable knowledge that public health efforts to improve breast cancer mortality through prevention, early detection, and treatment should center on non-Hispanic Black and Hispanic women in these southern hot spot counties.

\section{Acknowledgments}

Funding: None.

\section{Footnote}

Provenance and Peer Review: This article was commissioned by the editorial office, Translational Cancer Research. The article did not undergo external peer review.

Conflicts of Interest: All authors have completed the ICMJE uniform disclosure form (available at http://dx.doi. org/10.21037/tcr.2018.09.11). The authors have no conflicts of interest to declare.

Ethical Statement: The authors are accountable for all aspects of the work in ensuring that questions related to the accuracy or integrity of any part of the work are appropriately investigated and resolved.

Open Access Statement: This is an Open Access article distributed in accordance with the Creative Commons Attribution-NonCommercial-NoDerivs 4.0 International License (CC BY-NC-ND 4.0), which permits the noncommercial replication and distribution of the article with the strict proviso that no changes or edits are made and the original work is properly cited (including links to both the formal publication through the relevant DOI and the license). See: https://creativecommons.org/licenses/by-nc-nd/4.0/.

\section{References}

1. American Cancer Society: Cancer Facts \& Figures 2018 [Internet], 2017 [cited 2018 Aug 21] Available online: https://www.cancer.org/research/cancer-facts-statistics/allcancer-facts-figures/cancer-facts-figures-2018.html

2. Wheeler SB, Reeder-Hayes KE, Carey LA. Disparities in breast cancer treatment and outcomes: biological, social, and health system determinants and opportunities for research. Oncologist 2013;18:986-93.

3. Smigal C, Jemal A, Ward E, et al. Trends in breast cancer by race and ethnicity: update 2006. CA Cancer J Clin 2006;56:168-83.

4. Fedewa SA, Edge SB, Stewart AK, et al. Race and ethnicity are associated with delays in breast cancer treatment (20032006). J Health Care Poor Underserved 2011;22:128-41.

5. Reeder-Hayes KE, Wheeler SB, Baggett CD, et al. Influence of provider factors and race on uptake of breast cancer gene expression profiling. Cancer 2018;124:1743-51.

6. Hershman DL, Unger JM, Barlow WE, et al. Treatment quality and outcomes of African American versus white breast cancer patients: retrospective analysis of Southwest Oncology studies S8814/S8897. J Clin Oncol 2009;27:2157-62.

7. Bickell NA, Wang JJ, Oluwole S, et al. Missed opportunities: racial disparities in adjuvant breast cancer treatment. J Clin Oncol 2006;24:1357-62.

8. Wheeler SB, Carpenter WR, Peppercorn J, et al. Structural/organizational characteristics of health services partly explain racial variation in timeliness of radiation therapy among elderly breast cancer patients. Breast Cancer Res Treat 2012;133:333-45.

9. Camacho FT, Tan X, Alcalá HE, et al. Impact of patient race and geographical factors on initiation and adherence to adjuvant endocrine therapy in medicare breast cancer survivors. Medicine 2017;96:e7147.

10. Wheeler SB, Spencer JC, Pinheiro LC, et al. Financial impact of breast cancer in black versus white women. J Clin Oncol 2018;36:1695-701.

11. Harper S, Lynch J, Meersman SC, et al. Trends in areasocioeconomic and race-ethnic disparities in breast cancer incidence, stage at diagnosis, screening, mortality, and survival among women ages 50 years and over (1987-2005). Cancer Epidemiol Biomarkers Prev 2009;18:121-31.

12. Reyes-Ortiz CA, Eschbach K, Zhang DD, et al. Neighborhood composition and cancer among Hispanics: 
tumor stage and size at time of diagnosis. Cancer Epidemiol Biomarkers Prev 2008;17:2931-6.

13. Pruitt SL, Lee SJC, Tiro JA, et al. Residential racial segregation and mortality among black, white, and Hispanic urban breast cancer patients in Texas, 1995 to 2009. Cancer 2015;121:1845-55.

14. Pinheiro LC, Check DK, Rosenstein D, et al. Examining potential gaps in supportive medication use for US and foreign-born Hispanic women with breast cancer. Support Care Cancer 2018. [Epub ahead of print].

15. Peppercorn J, Horick N, Houck K, et al. Impact of the elimination of cost sharing for mammographic breast cancer screening among rural US women: A natural experiment. Cancer 2017;123:2506-15.

16. Peppercorn J, Houck K, Beri N, et al. Breast cancer screening utilization and understanding of current guidelines among rural U.S. women with private insurance. Breast Cancer Res Treat 2015;153:659-67.

17. Moss JL, Liu B, Feuer EJ. Urban/rural differences in breast and cervical cancer incidence: the mediating roles of socioeconomic status and provider density. Womens Health Issues 2017;27:683-91.

18. Williams F, Jeanetta S, James AS. Geographical location and stage of breast cancer diagnosis: A systematic review of the literature. J Health Care Poor Underserved 2016;27:1357-83.

19. Yao N, Alcalá HE, Anderson R, et al. Cancer disparities in rural appalachia: incidence, early detection, and survivorship. J Rural Health 2017;33:375-81.

Cite this article as: Ray EM, Wheeler SB, Reeder-Hayes KE. Race and place: complex constructs in breast cancer care. Transl Cancer Res 2018;7(6):1361-1365. doi: 10.21037/tcr.2018.09.11
20. Zahnd WE, Jenkins WD, Mueller-Luckey GS. Cancer Mortality in the Mississippi Delta Region: Descriptive Epidemiology and Needed Future Research and Interventions. J Health Care Poor Underserved 2017;28:315-28.

21. Keating NL, Landrum MB, Guadagnoli E, et al. Factors related to underuse of surveillance mammography among breast cancer survivors. J Clin Oncol 2006;24:85-94.

22. Enewold L, McGlynn KA, Zahm SH, et al. Surveillance mammography among female Department of Defense beneficiaries: a study by race and ethnicity. Cancer 2013;119:3531-8.

23. Lipscomb J, Gillespie TW, Goodman M, et al. Black-white differences in receipt and completion of adjuvant chemotherapy among breast cancer patients in a rural region of the US. Breast Cancer Res Treat 2012;133:285-96.

24. Moore JX, Royston KJ, Langston ME, et al. Mapping hot spots of breast cancer mortality in the United States: place matters for Blacks and Hispanics. Cancer Causes Control 2018. [Epub ahead of print].

25. Williams DR, Mohammed SA, Shields AE. Understanding and effectively addressing breast cancer in African American women: Unpacking the social context. Cancer 2016;122:2138-49.

26. Søgaard M, Thomsen RW, Bossen KS, et al. The impact of comorbidity on cancer survival: a review. Clin Epidemiol 2013;5:3-29. 